\title{
Fingerprint Image Enhancement Algorithms for Identification in an Electoral Process
}

\author{
Olabode, O., Akintola K.G
}

Computer Science Department

Fasoranbaku O.A

\section{Statistics Department}

The Federal University of Technology, Akure

\begin{abstract}
Electoral process in many developing nations is characterized with fraud and failure as a result of inefficient and effective system of voter's register and voting system. This usually leads to crises. This paper presents enhancement algorithms of Denoising fingerprint images using Moving Average Filter, the Segmentation of Fingerprint using Chen algorithm, Gradient-Based Local Ridge Orientation, Binarization using Otsu Algorithm, Thinning of Fingerprint Image using the linear interactive approach and Minutiae Extraction using the minutiae location and minutiae angles for the purpose of verification and Identification in electoral process. The input to the system is a Gray-scale Fingerprint image of electorate and it detailed information while the Output is a Verified and enrolled fingerprint image with matching score and details of electorates' information store as Template using the Matlab 2013a.
\end{abstract}

\section{Council for Innovative Research}

Peer Review Research Publishing System

Journal: INTERNATIONAL JOURNAL OF COMPUTERS \& TECHNOLOGY

Vol. 14, No. 2

www.ijctonline.com , editorijctonline@gmail.com 


\subsection{INTRODUCTION}

Voter registration is one of the stages at which there are ample opportunities to manipulate election results. Identification of electorate during elections is a great and challenging problem in most part of the developing countries of the world. In most cases, frauds are perpetrated because the mechanism put in place to reduce fraud during election is very weak. It is noted that the only means to identify an individual is through a voter's card. Identification of person can be compromised, multiple registration and voting can occur, imposter may be allowed to vote, voters card can be sold and so on. To develop a secure and effective voter's registration and control election systems requires personal-identification technologies that are reliable,implementable and convenient. In recent times Biometric based technology has enable the identification of individuals either by physical characteristics Nagendra, Sridhar, Prameelamma and Zubair (2012) such as finger prints, iris, finger vein, face or even hand geometry and the behavioral characteristics such as voice, signature and other typing pattern (Maltoni, Davide, Maio, Jain, and Prabhakar, 2009). They are used in broad range of systems for functions such as online banking, automatic teller machines for transaction, login for PC's, driver identification, libraries etc. In this study, an algorithm of secured fingerprint based registration and identification system for electorates is proposed.

According to Maltoni, Davide, Maio, Jain, and Prabhakar, (2009), biometrics is not only a fascinating pattern recognition research problem but, if carefully used, is an enabling technology with the potential to make our society safer, reduce fraud and provide user convenience (user friendly man-machine interface). The five most common classes of the Henry (1990) classification scheme includes arch, tented arch, left loop, right loop, and whorl

The Fingerprints are today the biometric features most widely used for personal identification. A fingerprint pattern is characterized Nagendra, Sridhar, Prameelamma and Zubair (2012) by a set of ridgelines that often flow in parallel, but intersect and terminate at some points. Minutiae characteristics are local discontinuities in the fingerprint pattern and represent the two most prominent local ridge characteristics: terminations and bifurcations. A ridge termination is defined as the point where a ridge ends abruptly, while ridge bifurcation is defined as the point where a ridge forks or diverges into branch ridges.

The uniqueness of a fingerprint is determined by their local ridge characteristics and their relationships. Most automatic systems for fingerprint comparison are based on minutiae matching. In electoral processes, fingerprint is the only accredited secured means of voting. Impression is made on ballot papers and this is only used as an indicator of choice of candidate. The voter's cards are merely inspected and this suffices for identification of voter which isassociated with fraud and failure. To ameliorate this ugly incidence, Effort should be put in place to harmonize the natural and simplified identification object and the information system. This calls for Fingerprint Image Enhancement Algorithm for registration and Identification in an Electoral Process.

\subsection{Electoral Process}

When a voter arrives at a polling unit, the voter present his voter's card. Electoral official is required by State law to determine whether the name on the ID matches the name on the list of registered voters, the voter will follow the regular procedures for voting. Voter will then be issued a ballot paper which he is required to thumb print to make choice of candidate. At the end of voting exercise, counting commences. At this juncture, fraud is inevitable. Multiple voting, impersonation, invalid votes, etc could occur deliberately. In this research work, we proposed an algorithm for the development of a secure and robust biometric based voter's registration system. The use of biometric automatic identification will curb multiple voting and provide secure and perfect identification of voters and provide a mechanism for verification of election material in case of doubt.

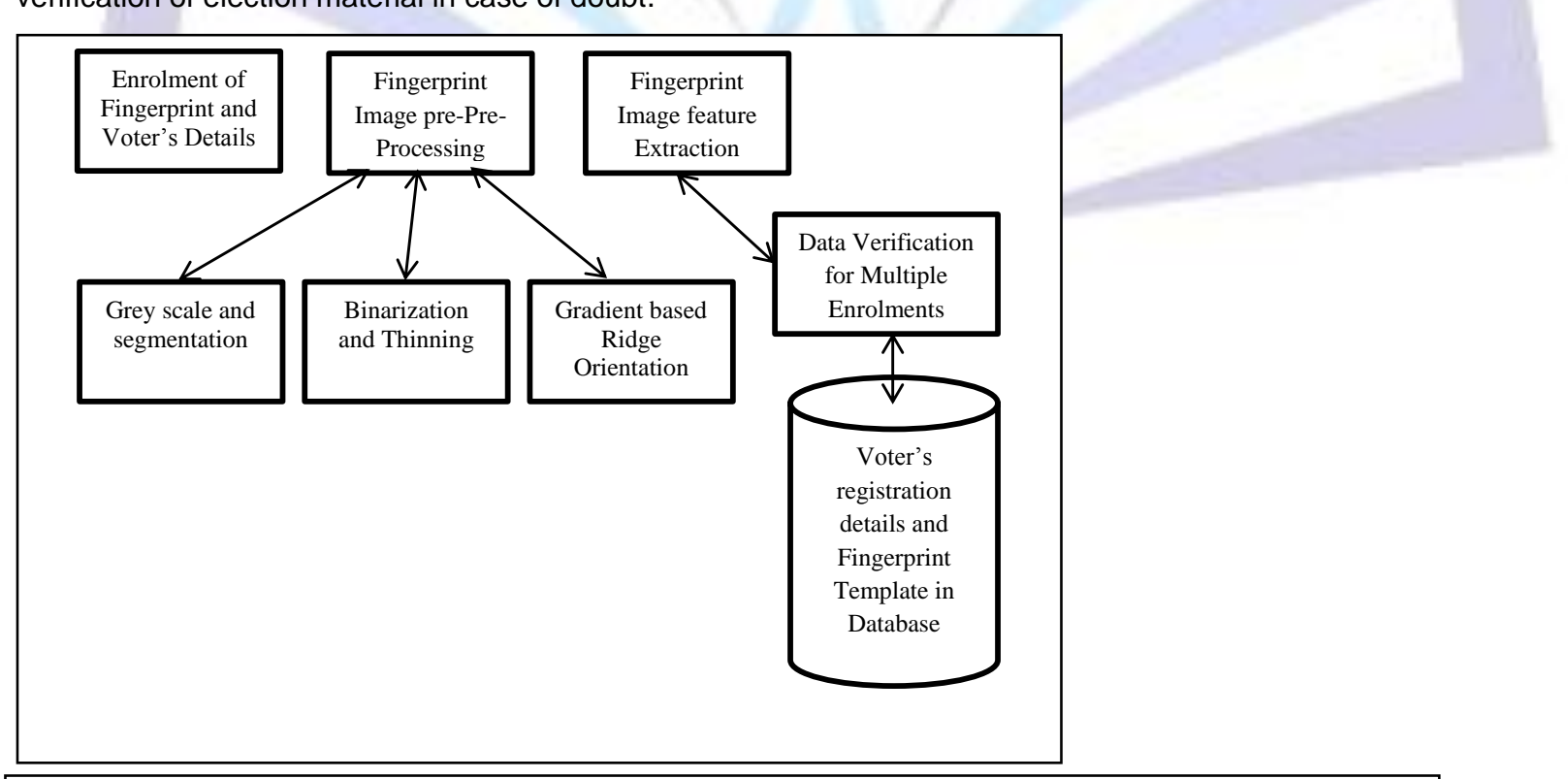

Figure 1: Architecture of the Biometric Based Voter Registration System. 
Figure 1 is a proposed architecture of the Biometric based voter registration system. The voter's details information to be captured into the database may include information on any citizen who has attained age of 18 years or more on the first day of January of the year. Detailed information in the form of database relation is

Electorate [Locality, Name,Date of Birth, Sex, Relation, Place of Ordinary Residence, Details of Family Members Already Included in the Electoral register, Declaration, Fingerprint]

The Image enhancement in electoral process is a preprocessing technique to make the fingerprint image clearer than the original image, for further operations (RajuRajkumar and K Hemachandran, 2011). Since the fingerprint images, acquired from sensors or other media are not always assured with perfect quality, in fingerprint recognition system, the enhancement methods are needed, to increase the contrast between ridges and furrows and for connecting the false broken points of ridges due to insufficient amount of ink etc.

\subsection{Process of Fingerprint Enhancement Model for Valid Voters Registration}

This session presentsenhancement algorithmsofDenoising fingerprint images using Moving Average Filter, the Segmentation of Fingerprint (Chan et. al, 2004), Gradient-Based Local Ridge Orientation (Ratha, chen, and Jain, 1995), Binarization (Otsu Algorithm), Thinning of Fingerprint Image (Zhang and Suen, 2010) and Minutiae Extraction using the minutiae location and minutiae angles for the purpose of Identification in electoral process. A digital image is generally encoded as a matrix of grayscale or color values. In the case of a movie, this matrix has three dimensions, the third one corresponding to time. Each pair $(\mathrm{i}, \mathrm{u}(\mathrm{i}))$, where $\mathrm{u}(\mathrm{i})$ is the value at $\mathrm{i}$, is called a pixel, short for "picture element." In the case of grayscale images, $i$ is a point on a two-dimensional (2D) grid and $u(i)$ is a real value. In the case of classical color images, $u(i)$ is a triplet of values for the red, green, and blue components (Buades, A. Coll B. and Morel J. M., 2010).

\subsection{De Noising Using Linear Filters in the spatial domain}

Most images are affected to some extent by noise that is unexplained variation in data, that is, disturbances in image intensity which are either uninterpretable or not of interest. Image analysis is often simplified if this noise can be filtered out. Employing the broader definition of filter in Glasbey, and Horgan, (1995), image filters may be used to emphasize edges, that is, boundaries between objects or parts of objects in images. Filters provide an aid to visual interpretation of images, and can also be used as a precursor to further digital processing, such as segmentation, thinning etc.

Definition: method noise.

Let $u$ be a (not necessarily noisy) image and $D_{h}$ a denoising operator depending on $h$. Then we define the method noise of $u$ as the image difference

$$
n\left(D_{h}, u\right)=u-D_{h}(u) \ldots 1
$$

Filters change a pixel's value taking into account the values of neighbouring pixels. They may either be applied directly to recorded images, or after transformation of pixel values. The Moving average applied in this work is a linear filter, because output values are linear combinations of the pixels in the original image. Moving average is an example of the linear spatial domain.

The moving average is the simplest of all Filters. It replaces each pixel by the average of pixel values in a square centered at that pixel. All linear filters work in the same way except that, instead of forming a simple average, a weighted average is formed. The algebra is as follows.

Let $f_{i j}$, for $i, j=1, \ldots, n$, denote the pixel values in the image at location $(i, j)$. For simplicity, images will be assumed to be square, with both the row index $(i)$ and the column index $(j)$ ranging between 1 and $n$. Then $g$, is taken with pixel values $g_{i j}$ to denote the output from the filter. A linear filter of size $(2 m+1) \times(2 m+1)$, with specified weights $w_{k l}$ for $k, l=$ $-m, \ldots, m$, gives

$$
g_{i j}=\sum_{k=-m}^{m} \sum_{l=-m}^{m} w_{k l} f_{i+k, j+l} \quad \text { for } i, j=(m+1), \ldots,(n-m) \quad \ldots 2
$$

For example, if $m=1$, then the window over which averaging is carried out is $3 \times 3$, and

$$
\begin{gathered}
g_{i j}=w_{-1,-1} f_{i-1, j-1}+w_{-1,0} f_{i-1, j}+w_{-1,1} f_{i-1, j+1} \\
+w_{0,-1} f_{-1, j-1}+w_{0,0} f_{i, j}+w_{0,1} f_{i, j+1} \\
\quad+w_{1,-1} f_{i+1, j-1}+w_{1,0} f_{i+1, j}+w_{1,1} f_{i+1, j+1}
\end{gathered}
$$

For full generality, the weights $(w)$ can depend on $i$ and $j$, resulting in a filter which varies across the image. However, the linear filters in a spatially invariant is considered in this work.

For the moving average filter, $w_{k l}=\frac{1}{(2 m+1)^{2}}$, the bigger the window the greater the noise reduction and blurring.Computational efficiency is an important consideration in image analysis because of the size of data sets(Glasbey, and Horgan, 1995). In total, there are $(2 m+1)^{2}$ additions and multiplications per pixel involved in deriving $\mathrm{g}$ from $f$. However, some filters can be computed more quickly. A filter is said to be separable if it can be performed by first filtering the image inside a $(2 m+1) \times 1$ window, and then inside a $1 \times(2 m+1)$ window. In other words, it can be separated into a column operation given by: 


$$
h_{i j}=\sum_{k=-m}^{m} w_{k}^{c} f_{i+k, j} \quad \text { for } i=(m+1), \ldots,(n-m) ; j=1, \ldots, n \quad \ldots 4
$$

using column weights $w_{-m}^{c}, \ldots, w_{m}^{c}$, followed by a row operation:

$$
g_{i j}=\sum_{l=-m}^{m} w_{l}^{r} h_{i, j+l} \quad \text { for } i, j=(m+1), \ldots,(n-m) \quad \ldots 5
$$

using row weights $w_{-m}^{r}, \ldots, w_{m}^{r}$.

In order for this to be possible, the array of weights, $w_{k l}$, must be expressible as the product of the column and row weights, as follows:

$$
w_{k l}=w_{k}^{c} w_{l}^{r} \quad \text { for } k, l=-m, \ldots, m
$$

The number of operations per pixel has been reduced from $(2 m+1)^{2}$ to $2(2 m+1)$. Therefore a separable filter can be computed more quickly than one that is not separable, even when

$\mathrm{m}=1$.

\subsection{The Segmentation of Fingerprint using the Chan et. al (2004)}

As presented in Chen et. al(2004), the segmentation of fingerprint algorithm partitioned a fingerprint into blocks of $w \times w$ pixels ( $w=12$ in Chens algorithm).

The segmentation of fingerprint images using linear classifier algorithm Chen et. al,(2004) uses three block features, being the block clusters degree, the block mean information, and the block variance. An optimal linear classifier could be employed for training and classification per block and the criteria of minimal number of misclassified samples are used. Morphological postprocessing is applied to reduce classification errors.

The feature extraction is to acquire a group of most optimal features for classification. In Chen a given criterion for evaluating a feature which is the classification error rate of the feature given as:

$$
E r r=\frac{N_{\text {err }}}{N_{\text {total }}}=p\left(\omega_{0} \mid \omega_{1}\right)+p\left(\omega_{1} \mid \omega_{0}\right)
$$

where $\omega_{0}$ represent background class, while $\omega_{1}$ represent foreground class

\section{The block clusters degree (CluD)}

The block clusters degree CluD measures how well the ridge pixels are clustering. Using $I$ as the intensity of image, the block clusters degree is defined as follows

$$
\begin{gathered}
\text { CluD }=\sum_{i, j \in \text { Block }} \operatorname{sign}\left(I_{i j}, I_{\text {mean }}\right) \cdot \operatorname{sign}\left(D_{i j}, \text { Thre }_{\text {CluD }}\right) \\
\text { where } D_{i j}=\sum_{m=i-2}^{i+2} \sum_{n=j-2}^{j+2} \operatorname{sign}\left(I_{m n}, I_{m g} g_{\text {mean }}\right) \\
\operatorname{sign}(\alpha, \beta)= \begin{cases}1 & \text { if }(\alpha<\beta) \\
0 & \text { otherwise }\end{cases}
\end{gathered}
$$

$I m g_{\text {mean }}$ is the intensity mean of the whole image. While $D_{i j}$ is computed using the pixel centered $p_{i j}$. ThreCluD is an empirical parameter which could be any numerical value in the range of 2 to 15.

\section{The block mean information (Meanl)}

The mean information Meanl for each block is given by

$$
\text { MeanI }=\left(\frac{1}{w \cdot w} \sum_{\text {Block }} I\right)-I m g_{\text {mean }}
$$

\section{The block variance (Varl)}

The block variance Var is the third feature that is used. In general, the variance of the ridge-valley structures in the foreground is higher than the variance of the noise in the background (See Figure 2). The block variance Var for each block is given by 


$$
\operatorname{Var}=\frac{1}{w \cdot w} \sum_{B l o c k}(I-m e a n)^{2}
$$

\section{Linear classification design}

In Chen et. al (2004), a classification algorithm was constructed that minimizes the probability of misclassifying, because it is very important to use a classification algorithm that has the lowest computational complexity possible. Therefore a linear classifier satisfying the condition is given by

$$
v=w^{T} x=w_{0} C l u D+w_{1} \text { MeanI }+w_{2} \operatorname{Var}+w_{3}
$$

where $v$ is the value to be tested, $w=\left[w_{0} w_{1} w_{2} w_{3}\right]^{T}$ is the weight vector, and

$x=[\mathrm{CluD}+\mathrm{MeanI}+\mathrm{Var}]^{T}$ is the feature vector. Then, using class $\omega_{1}$ for the foreground, class $\omega_{0}$ for the background, and $\widehat{\omega}$ for the assigned class, the following decision function is applied

$$
\widehat{\omega}= \begin{cases}\omega_{1} & \text { if } w^{T} x>0 \\ \omega_{0} & \text { otherwise }\end{cases}
$$

If the samples are two linearly separable classes, we know that there exists a vector $w$, satisfying

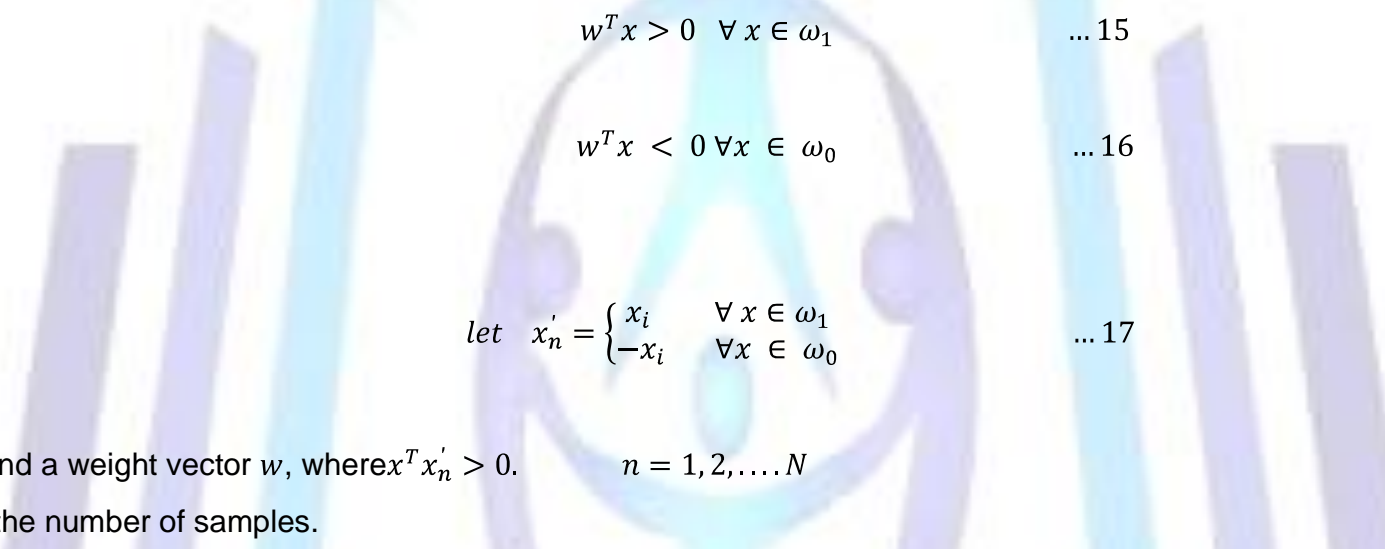

then to find a weight vector $w$,
and $N$ is the number of samples.

The next phase of the Chen et. al (2004) algorithm is to apply morphology to the classification estimate. This reduces the number of false classifications. First, small clusters that are incorrectly assigned to the foreground are removed by means of an open operation. Next, small clusters that are incorrectly assigned to the background are removed by a close operation. After the morphological processing, the edges and corners are then connected using the lines.

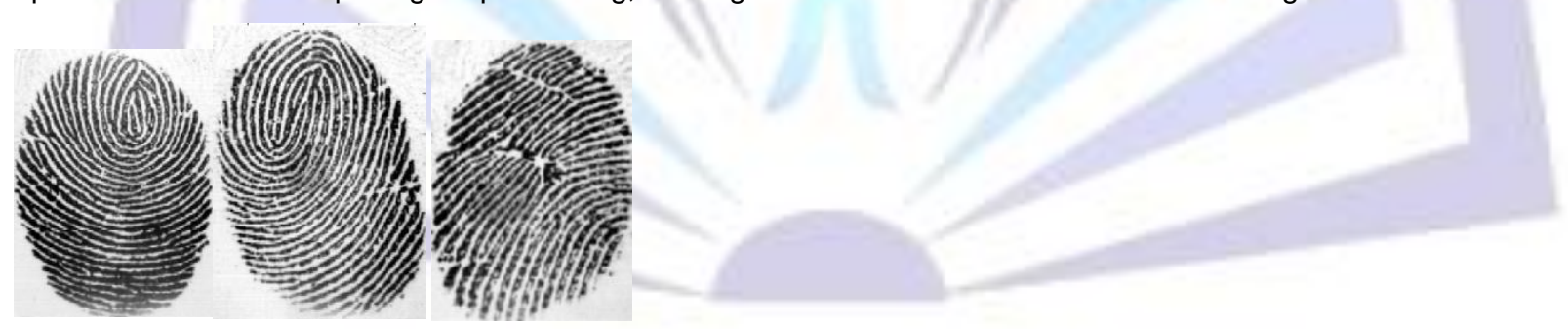

Figure 2: Denoised Fingerprint Image

\subsection{Otsu Algorithm for Binarization}

The operation that converts a gray-scale image into a binary image is known as binarization.Pixels with the value 0 are displayed as black; pixels with the value 1 are displayed as white (See Figure 3).

Suppose the intensity of a gray level image be expressed in $L$ gray levels $[1,2, \ldots,, L]$. The number of points with gray level at $i$ is denoted by $x_{i}$ and the entire number of points can be expressed as $=x_{1}+x_{2}+\ldots+x_{L}$. The histogram of this graylevel image is regarded as an occurrence distribution of probability (Xiaolu, Xuanjing, Jianwu and Haipeng (2012).

$$
p(i)=\frac{x_{i}}{X}, \quad x_{i} \geq 0 . \quad \sum_{i=1}^{L} x_{i}=1
$$


The image pixels are divided into two parts $C_{0}$ and $C_{1}$, i.e. foreground and background by a threshold $t$. Where $C_{0}$ represents pixels within levels $[1,2, \ldots, t]$ ans $C_{1}$ denotes pixels within $[t+1, t+2, \ldots, L]$. The occurrence probabilities of this class and average can be expressed respectivelyas:

$$
\begin{aligned}
& \omega_{0}=\omega(t)=\sum_{i=1}^{t} p(i) \\
& \omega_{1}=1-\omega(t)=\sum_{i=t+1}^{L} p(i) \\
& \mu_{0}=\sum_{i=1}^{t} \frac{i \cdot p(i)}{\omega_{0}}=\frac{1}{\omega(t)} \sum_{i=1}^{t} p(i) \\
& \mu_{1}=\sum_{i=t+1}^{L} \frac{i \cdot p(i)}{\omega_{1}}=\frac{1}{1-\omega(t)} \sum_{i=t+1}^{L} i \cdot p(i)
\end{aligned}
$$

Total mean can be written as

$$
\mu_{T}=\sum_{i=1}^{L} i \cdot p(i)
$$

And then compute for $\mu_{T}=\omega_{0} \mu_{0}+\omega_{1} \mu_{1}$

Where $\omega_{0}$ and $\omega_{1}$ denote probability of foreground part and background part respectively. Besides $\mu_{0}, \mu_{1}$, and $\mu_{T}$ refer to the mean in grey levels of the foreground of the gray image, the background of the gray image and the entire gray level image.

The between-class variance $\sigma_{B}^{2}$ of the two classes $C_{0}$ and $C_{1}$ is given by

$$
\sigma_{B}^{2}=\omega_{0}\left(\mu_{0}-\mu_{T}\right)^{2}+\omega_{1}\left(\mu_{1}-\mu_{T}\right)^{2}
$$

The separable degree $\eta$ of the class, in the discrimination, is $\eta=\max _{1 \leq t \leq L} \sigma_{B}^{2}$

Finally, maximizing $\sigma_{B}^{2}$ to choose the optimal threshold $t^{*} . \quad t^{*}=\arg \max _{1 \leq t \leq L} \sigma_{B}^{2}$
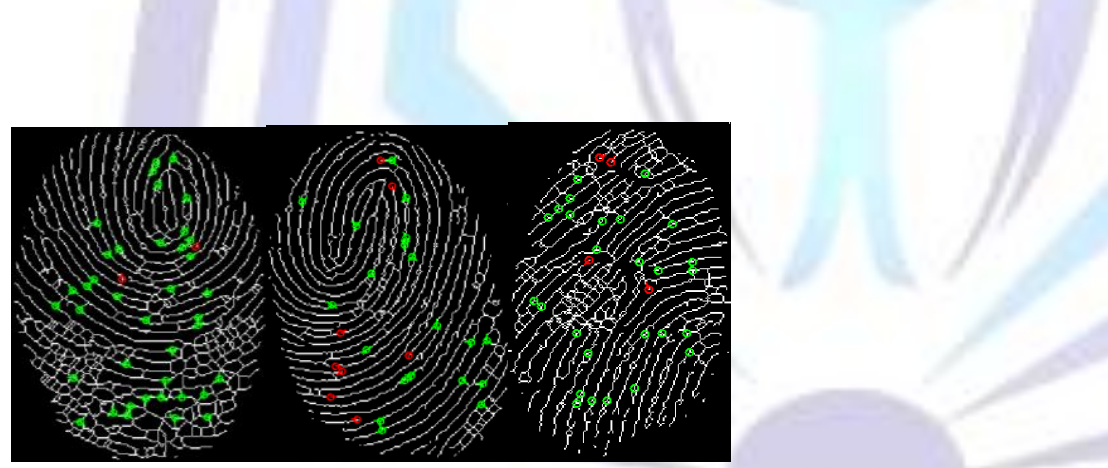

Figure 3: A gray-scale image or binarization Image with Pixels value 0 as black and pixels value 1 as white

\subsection{Gradient-Based Local Ridge Orientation}

The local ridge orientation at a pixel $[x, y]$ is the angle $\theta_{x y}$ that the fingerprint ridges, crossing through an arbitrary small neighborhood centered at $[x, y]$, form with the horizontal axis. The simplest and most natural approach for extracting local ridge orientation is based on computation of gradients in the fingerprint image. The gradient $\nabla(x, y)$ at point $[x, y]$ of $I$, is a two dimensional vector $[\nabla x(x, y), \nabla y(x, y)]$, where $\nabla x$ and $\nabla y$ components are the derivatives of $I$ at $[x, y]$ with respect to the $x$ and $y$ directions, respectively.

Ratha, Chen, and Jain (1995) computed the dominant ridge orientation $\theta_{i j}$ by combining multiple gradient estimates within a $17 \times 17$ window $W$ centered at $x_{i}, y_{j}$

$$
\begin{aligned}
& \theta_{i j}=90^{\circ}+\frac{1}{2} \operatorname{atan} 2\left(2 G_{x y}, G_{x x}-G_{x y}\right) \\
& G_{x y}=\sum_{h=-8}^{8} \sum_{k=-8}^{8} \nabla_{x}\left(x_{i}+h, y_{j}+k\right) \cdot \nabla_{y}\left(x_{i}+h, y_{j}+k\right) \ldots 26
\end{aligned}
$$




$$
\begin{aligned}
& G_{x x}=\sum_{h=-8}^{8} \sum_{k=-8}^{8} \nabla_{x}\left(x_{i}+h, y_{j}+k\right)^{2} \\
& G_{y y}=\sum_{h=-8}^{8} \sum_{k=-8}^{8} \nabla_{y}\left(x_{i}+h, y_{j}+k\right)^{2}
\end{aligned}
$$

where $\nabla x$ and $\nabla y$ are the $x$ - and $y$-gradient components computed through $3 \times 3$ Sobel masks, and atan2( $y, x)$ calculates the arctangent of the two variables $y$ and $x$ : it is similar to calculating the arctangent of $y / x$, except that the signs of both arguments are used to determine the quadrant of the result.

The reliability $r$ of the estimate or the local coherence map is computed using:

$$
r_{i j}=\operatorname{coherence}\left(\theta_{i j}\right)=\frac{\sqrt{\left(G_{x x}-G_{y y}\right)^{2}+4 G_{x y}^{2}}}{G_{x x}+G_{y y}} \quad \ldots 30
$$

Jain et al. (1997) computed the concordance of the orientations according to their variance in $5 \times 5$ neighborhoods whereas Donahue and Rokhlin (1993) computed this according to the residual of the least-square minimization.

\subsection{Thinning Algorithm for Fingerprint Image}

Skeletonization or thinning operation can be done by iteratively eroding away nonessential pixels from the image until one is left with a stick figure, or skeleton, which describes the object. Skeletonization algorithms are the need to compute a reduced amount of data or to simplify the shape of an object in order to find features for recognition algorithms and classifications. In iterative methods, thinning algorithms produce a skeleton by examining and deleting contour pixels through an iterative process in either sequential or parallel way. Ashwiniand Navalgunda (2013) opine that the ZhangSuen's thinning algorithm is an iterative algorithm. It is fast and simple to be implemented. In the algorithm it is assumed that the region point in the image has pixel value ' 1 ' and background points have value ' 0 '.

In Zhang and Suen, (2010), A binary digitized picture is defined by a matrix ITwhere each pixel IT $(i, j)$ is either 1 or 0. The pattern consists of those pixels that have value 1. Each stroke in the pattern is more than one element thick. Iterative transformations are applied to matrix IT point by point according to the values of a small set of neighboring points. It is assumed that the neighbors of the point $(i, j)$ are

$(i-1, j),(i-1, j+1),(i, j+1),(i+1, j+1),(i+1, j),(i+1, j-1),(i, j-1)$, and $(i-1, j-1)$, as is shown in Figure 1 . In parallel picture processing, the new value given to a point at the nth iteration depends on its own value as well as those of its eight neighbors at the $(n-1)$ th iteration, so that all picture points can be processed simultaneously. It is assumed that a $3 \times 3$ window is used, and that each element is connected with its eight neighboring elements.

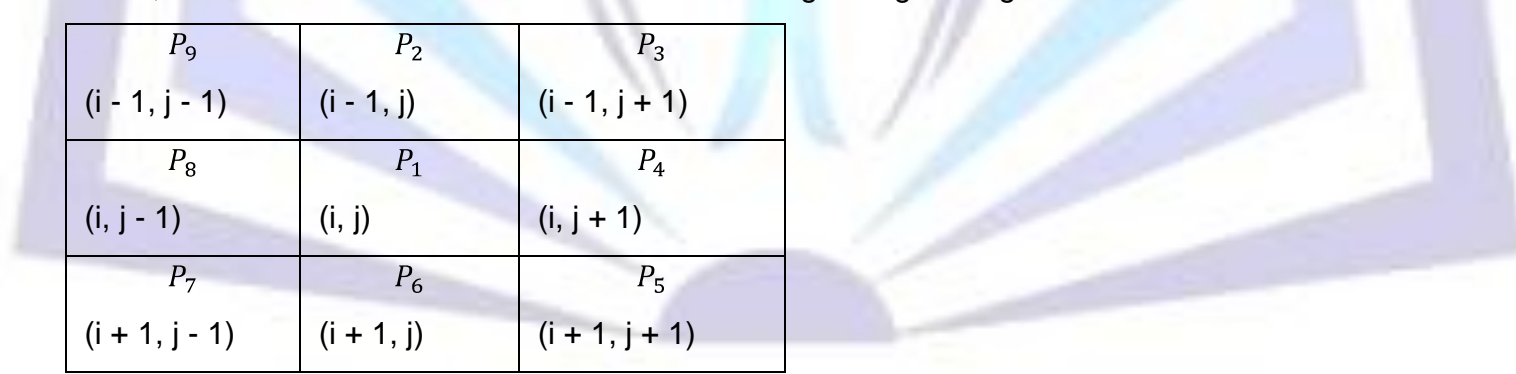

Figure 4: Designations of the nine pixels in a $3 \times 3$ window

The method for extracting the skeleton of a picture consists of removing all the contour points of the picture except those points that belong to the skeleton. In order to preserve the connectivity of the skeleton, divide each iteration into two subiterations. In the first subiteration, the contour point $P_{1}$ is deleted from the digital pattern if it satisfies the following conditions (Figure 7):
(a) $2 \leq B\left(P_{1}\right) \leq 6$
(b) $A\left(P_{1}\right)=1$
(c) $P_{2} * P_{4} * P_{6}=0$
(d) $P_{4} * P_{6} * P_{8}=0$

where $A\left(P_{1}\right)$ is the number of 01 patterns in the ordered set $P_{2}, P_{3}, P_{4}, \ldots, P_{8}, P_{9}$ that are the eight neighbors of $P_{1}($ Figure 4$)$, and $B\left(P_{1}\right)$ is the number of nonzero neighbors of $P_{1}$, that is,

$B\left(P_{1}\right)=P_{2},+P_{3}+P_{4}+\ldots+P_{8}+P_{9}$. 
If any condition is not satisfied, e.g., the values $P_{2}, P_{3}, P_{4}, \ldots, P_{8}, P_{9}$ as shown in Figure 5 , then $A\left(P_{1}\right)=2$. Therefore, $P_{1}$ is not deleted from the picture.

In the second subiteration, only conditions (c) and (d) are changed (Figure 6) as follows:

(c') $P_{2} * P_{4} * P_{8}=0$

(d') $P_{2} * P_{6} * P_{8}=0$ and the rest remain the same.

By conditions (c) and (d) of the first subiteration, it will be shown that the first subiteration removes only the south-east boundary points and the north-west corner points which do not belong to an ideal skeleton.

\begin{tabular}{|l|ll|l|}
\hline 0 & 0 & & 1 \\
\hline 1 & & $P_{1}$ & 0 \\
\hline 1 & 0 & & 0 \\
\hline \multicolumn{3}{|c|}{$(2)$} \\
\hline
\end{tabular}

Figure 5 Counting the 01 patterns in the ordered set $P_{2}, P_{3}, P_{4}, \ldots, P_{8}, P_{9}$

North

West

\begin{tabular}{|c|c|c|}
\hline & $P_{2}$ & \\
\hline$P_{8}$ & $P_{1}$ & $P_{4}$ \\
\hline & $P_{6}$ & \\
\hline
\end{tabular}

Figure 6 Points under consideration and their locations

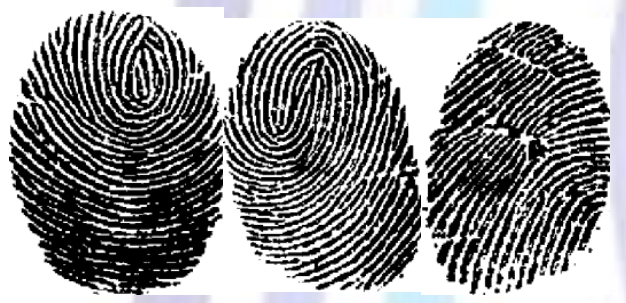

Figure 7: SkeletonizationFingerprint Image after Denoising and Binarization operations

\section{Feature Extraction}

Minutiae Matching: To compare the input fingerprint data with the template data Minutiae matching is used. For efficient matching process, the extracted data is stored in the matrix format. The data matrix is as follows.

Number of rows: Number of minutiae points.

Number of columns: 4

Column 1: Row index of each minutia point.

Column 2: Column index of each minutia point.

Column 3: Orientation angle of each minutia point.

Column 4: Type of minutia. (A value of ' 1 ' is assigned for termination, and ' 3 ' is assigned for bifurcation).

During the matching process, each input minutiae point is compared with template minutiae point. In each case, template and input minutiae are selected as reference points for their respective data sets. The reference points are used to convert the remaining data points to polar coordinates. The Equation (31) is used to convert the template minutiae from row and column indices to polar coordinates

$$
\left[\begin{array}{c}
r_{k}^{T} \\
\phi_{k}^{T} \\
\theta_{k}^{T}
\end{array}\right]=\left[\begin{array}{c}
\sqrt{\left(\operatorname{row}_{k}^{T}-\operatorname{row}_{r e f}^{T}\right)^{2}+\left(\operatorname{col}_{k}^{T}-\operatorname{col}_{r e f}^{T}\right)^{2}} \\
\tan ^{-1}\left(\frac{\operatorname{row}_{k}^{T}-\operatorname{row}_{r e f}^{T}}{\operatorname{col}_{k}^{T}-\operatorname{col}_{r e f}^{T}}\right) \\
\theta_{k}^{T}-\theta_{r e f}^{T}
\end{array}\right]
$$

Where, for a template image,

$r_{k}^{T}=$ radial distance of kth minutiae. 
$\phi_{k}^{T}=$ radial angle of kth minutiae.

$\theta_{k}^{T}=$ orientation angle of $\mathrm{k}$ th minutiae.

row $_{\text {ref }}^{T}, \operatorname{col}_{\text {ref }}^{T}=$ row index and column index of reference points currently being considered.

Similarly the input matrix data points are converted to polar coordinates using the Equation (32)

$$
\left[\begin{array}{c}
r_{k}^{I} \\
\phi_{k}^{T} \\
\vartheta_{k}^{T}
\end{array}\right]=\left[\begin{array}{c}
\sqrt{\left(\operatorname{row}_{m}^{I}-\operatorname{row}_{r e f}^{I}\right)^{2}+\left(\operatorname{col}_{m}^{I}-\operatorname{col}_{r e f}^{I}\right)^{2}} \\
\tan ^{-1}\left(\frac{\operatorname{row}_{m}^{I}-\operatorname{row}_{r e f}^{I}}{\operatorname{col}_{k}^{I}-\operatorname{col}_{r e f}^{I}}\right)+\operatorname{rotatevalue}(k, m) \\
\theta_{m}^{I}-\theta_{r e f}^{I}
\end{array}\right]
$$

Rotate values $(k, m)$ represents the difference between the orientation angles of $T k$ and $I m$. $T k$ and $I m$ represent the extracted data in all the columns of row $k$ and row $m$ in the template and input matrices, respectively.

Minutiae Extraction: The minutiae location and the minutiae angles are derived after minutiae extraction. The terminations which lie at the outer boundaries are not considered as minutiae points, and Crossing Number is used to locate the minutiae points in fingerprint image. Crossing Number is defined as half of the sum of differences between intensity values of two adjacent pixels. If crossing Number is 1,2 and 3 or greater than 3 then minutiae points are classified as Termination, Normal ridge and Bifurcation respectively

Terminations :

$\begin{array}{ccc}X & Y & \text { Angle } \\ 161 & 33 & 169.00 \\ 36 & 147 & 98.00 \\ 154 & 103 & 205.00 \\ 103 & 175 & 110.00 \\ 195 & 123 & 132.00\end{array}$

Bifurcations :

\begin{tabular}{lrccc}
\hline$X$ & $Y$ & Angle 1 & Angle 2 & Angle 3 \\
192 & 44 & 198.00 & 45.00 & 146.00 \\
48 & 141 & 61.00 & 133.00 & 68.00 \\
202 & 69 & 157.00 & 70.00 & 141.00 \\
72 & 126 & 74.00 & 206.00 & 74.00 \\
175 & 75 & 163.00 & 76.00 & 211.00 \\
78 & 159 & 96.00 & 188.00 & 104.00 \\
225 & 104 & 201.00 & 110.00 & 225.00 \\
110 & 147 & 113.00 & 116.00 & 131.00 \\
121 & 135 & 230.00 & 143.00 & 208.00 \\
148 & 145 & 153.00 & 204.00 & 153.00 \\
221 & 153 & 192.00 & 154.00 & 230.00 \\
158 & 223 & 166.00 & 153.00 & 167.00 \\
166 & 178 & 185.00 & 191.00 & 141.00 \\
193 & 148 & 195.00 & 156.00 & 199.00 \\
166 & 199 & 145.00 & 201.00 & 164.00
\end{tabular}




\section{Process of Identification in Electoral Process}

In identification during registration, system recognizes an individual by searching the templates of all the users in the database for a match. Therefore, the system conducts a one-to-many comparison to establish an individual's identity (or fails if the subject is not enrolled in the system database) without the subject having to claim an identity. Identification is a critical component in negative recognition applications where the system establishes whether the person is who she (implicitly or explicitly) denies to be. The purpose of negative recognition is to prevent a single person from using multiple identities. Identification may also be used in positive recognition for convenience (the user is not required to claim an identity).

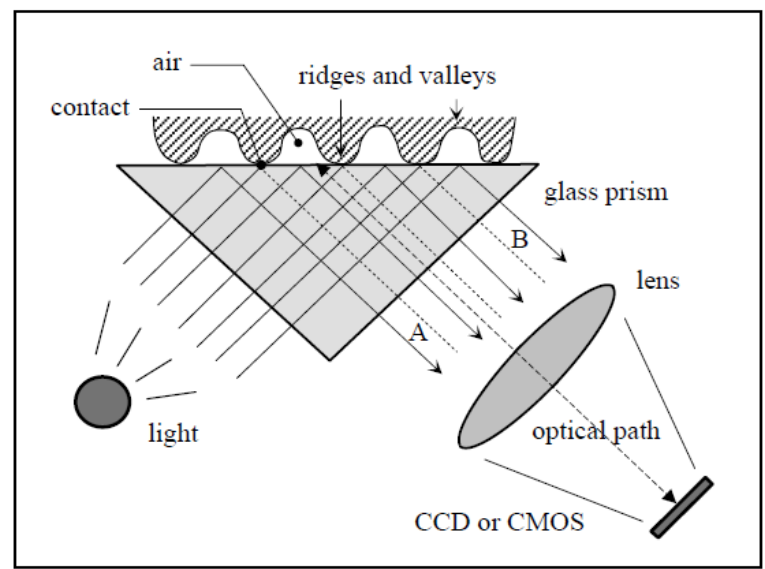

Figure 8: FTIR -Based Fingerprint Sensor Operation

Figure 3 shows the Frustrated Total Internal Reflection (FTIR) which is the oldest and most commonly used live-scan acquisition technique today (Hase and Shimisu (1984); Bahuguna and Corboline (1996)). As the finger touches the top side of a glass/plastic prism, the ridges are in optical contact with the prism surface, but the valleys remain at a certain distance. The left side of the prism is typically illuminated through a diffused light (a bank of light-emitting diodes [LEDs] or a film of planar light). The light entering the prism is reflected at the valleys, and randomly scattered (absorbed) at the ridges.

\section{The Algorithms for Voter registration and Identification system}

At the stage of enrolment, the fingerprint images is captured and shown in Figure 8 and pre-processed using enhancement algorithms as presented below

a. The Fingerprint Image EnrolmentInput: Gray-scale Fingerprint image and electorate detailed information.

b. Figerprint is Denoise using Moving Average linear filter in Spatial domain, then Segmentation of Fingerprint by Chan et. al, 2004)

c. Binarization using Otsu Algorithm

d. Local ridge orientation is computed using the Gradient-Based Local Ridge Orientation (Ratha, chen, and Jain, 1995)

e. $\quad$ Thinning of Fingerprint Image (Zhang and Suen, 2010)

f. Feature Extraction using the minutiae location and minutiae angles. Minutiae points are extracted. Data matrix is generated to get the position, orientation and type of minutiae.

g. Matching score of enrolled images is computed, and compared with Matching scores in the database. If matching score is 1 images are matched, meaning candidate already registered and if it is 0 then they are mismatched, then details of candidate can then be stored.Features are extracted and stored in a database. Thereafter, identification can proceed during election.

Output: Verified fingerprint image with matching score and details of electorates information store as Template using the Matlab 2013a.

\section{Conclusion}

Elcetorial process in many developing nations is characterized with fraud and failure and this in most cases leads to crises. This paper presents enhancement algorithms of fingerprint images Denoising using Moving Average Filter, the Segmentation of Fingerprint (Chan et. al, 2004), Gradient-Based Local Ridge Orientation (Ratha, chen, and Jain, 1995), Binarization (Otsu Algorithm), Thinning of Fingerprint Image (Zhang and Suen, 2010) and Minutiae Extraction using the minutiae location and minutiae angles for the purpose of verification and Identification in electoral process. The input to the system is a Gray-scale Fingerprint image of electorate and it detailed information while the Output is a Verified and enrolled fingerprint image with matching score and details of electorates information store as Template using the Matlab $2013 a$.

The uniqueness of a fingerprint is determined by their local ridge characteristics and their relationships. A fingerprint pattern is characterized by a set of ridgelines that often flow in parallel, but intersect and terminate at some points. Minutiae characteristics are local discontinuities in the fingerprint pattern and represent the two most prominent local ridge 
characteristics: terminations and bifurcations. A ridge termination is defined as the point where a ridge ends abruptly, while ridge bifurcation is defined as the point where a ridge forks or diverges into branch ridges.

In electoral processes, fingerprint is the only accredited secured means of voting. Impression is made on ballot papers and this is only used as an indicator of choice of candidate. In recent elections, voter's cards are merely inspected and this suffices for identification of voter which is associated with fraud and failure. To ameliorate this ugly incidence, Effort should be put in place to harmonize the natural and simplified identification object and the information system. This calls for Fingerprint Image Enhancement Algorithm for registration and Identification in an Electoral Process.

\section{Reference}

1. Ashwini S. Karnea and S. S. Navalgunda (2013). Implementation of an Image Thinning Algorithm using Verilog and MATLAB. International Journal of Current Engineering and Technology. Proceedings of National Conference on 'Women in Science \& Engineering' (NCWSE 2013), SDMCET Dharwad. ISSN 2277 - 4106.

2. Bahuguna R.D. and Corboline T., (1996). Prism fingerprint sensor that uses a holographic element. Applied Optics, vol. 35, no. 26, pp. 5242-5245, 1996.

3. Buades, A. Coll B. and Morel J. M. (2010). Image Denoising Methods. A New Nonlocal Principle SIAM REVIEW, Vol. 52, No. 1, pp. 113-147, 2010 Society for Industrial and Applied Mathematics

4. Chan T. and Vese, L. (2001). Active contours without edges. in IEEE transactions on image processing 10(2), 2001, pp. 266-277.

5. Chen X., Tian J., Cheng J. and Yang X.,(2004).Segmentation of fingerprint images using linear classifier. EURASIP Journal on Applied Signal Processing, vol. 2004, no. 4, pp. 480-494, 2004.

6. Donahue and Rokhlin (1993). Donahue M.L and Rokhlin S.I., "On the use of level curves in image analysis," CVGIP: Image Understanding, vol. 57, no. 2, pp. 185-203, 1993.

7. Glasbey, C. A. and Horgan, G. W. (1995). Image Analysis for the Biological Sciences. John Wiley and Sons

8. Hase M. and Shimisu A.,(1984). Entry method of fingerprint image using a prism, Trans. Institute Electron. Commum. Eng. Jpn., vol. J67-D, pp. 627-628, 1984.

9. Henry E (1990). Classification and Uses of Finger Prints, Routledge, London.

10. Maltoni, Davide, Maio, Jain, and Prabhakar (2009). Handbook of Fingerprint Recognition. $2^{\text {nd }}$ Eds. Springer: New York.

11. Raju,Rajkumar and Hemachandran,K (2011). Assam University Journal of Science \& Technology. Physical Sciences and Technology. Vol. 7 Number II. 52-57. ISSN 0975-2773

12. Ratha, N.K., Chen, S.Y. and Jain, A.K. (1995). Adaptive flow orientation-based feature extraction in fingerprint images. Pattern Recognition, vol. 28, no. 11, pp. 1657-1672, 1995.

13. Xiaolu Yang, XuanjingShen, Jianwu Long and Haipeng Chen (2012). An Improved Median-based Otsu Image Thresholding Algorithm, AASRI Procedia 3 pp468 - 473

14. Zhang, T. Y. and Suen, C. Y. (1984). A Fast Parallel Algorithm for Thinning Digital Patterns. Image Processing and Computer Vision: Communications of the ACM 27(3) 